\title{
Data report: particle size distribution for IODP Expedition 329 sites in the South Pacific Gyre ${ }^{1}$
}

\author{
N. Dubois, ${ }^{2,3}$ N.C. Mitchell, ${ }^{2}$ and I.R. Hall ${ }^{4}$
}

\section{Chapter contents}

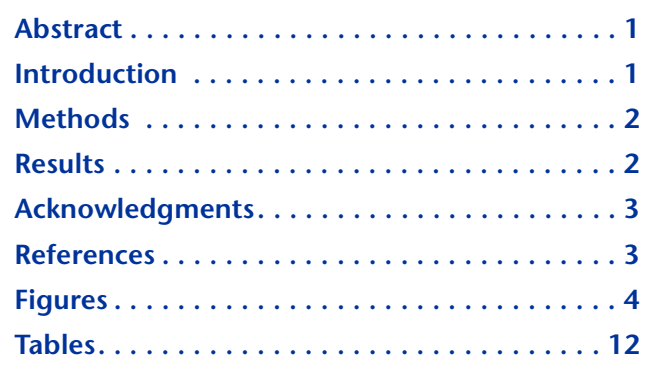

'Dubois, N., Mitchell, N.C., and Hall, I.R., 2014. Data report: particle size distribution for IODP Expedition 329 sites in the South Pacific Gyre. In D'Hondt, S., Inagaki, F., Alvarez Zarikian, C.A., and the Expedition 329 Scientists, Proc. IODP, 329: Tokyo (Integrated Ocean Drilling Program Management International, Inc.). doi:10.2204/iodp.proc.329.201.2014

${ }^{2} S$ chool of Earth, Atmospheric, and Environmental Sciences, The University of Manchester, Williamson Building (2.42), Oxford Road, Manchester M13 9PL, United Kingdom. Correspondence author: nathalie.dubois@eawag.ch

${ }^{3}$ Department of Geology and Geophysics, Woods Hole Oceanographic Institution, Woods Hole MA 02543, USA.

${ }^{4}$ School of Earth and Ocean Sciences, Cardiff University, Cardiff CF10 3YE, United Kingdom.

\section{Abstract}

We conducted particle size analyses on 208 samples from Integrated Ocean Drilling Program Expedition 329 in the South Pacific Gyre. Expedition 329 cored deep-sea sediment at seven sites along two transects in the center of the gyre. We analyzed the particle size distribution of sediment in the clay to silt size range $(1.5-63 \mu \mathrm{m})$ at the seven drill sites using a Sedigraph particle size analyzer. Here we describe our laboratory procedure and present the overall results. The majority of samples are composed of claysized material, with some samples having $>95 \mathrm{wt} \%$ of particles finer than $1.5 \mu \mathrm{m}$. However, a number of samples, in particular from sites located further offshore, show an extraordinarily well sorted particle size distribution.

\section{Introduction}

Integrated Ocean Drilling Program (IODP) Expedition 329 was conducted to investigate the subseafloor microbiology of the South Pacific Gyre. Its primary aim was to document the extent and character of life in sedimentary habitats with very low biomass and rates of activity (see the "Expedition 329 summary" chapter [Expedition 329 Scientists, 2011]). Although Expedition 329 focused on microbiology, the recovered cores also provide a unique opportunity to document a sedimentary system that has never been explored by scientific ocean drilling. The R/V JOIDES Resolution cored deep-sea sediment at seven sites (Fig. F1) located along two transects in the center of the South Pacific Gyre and spanning nearly the entire width of the Pacific plate in the Southern Hemisphere. The first transect progresses from the western edge of the gyre (Site U1365) to the gyre center (Site U1368) at $\sim 26^{\circ} \mathrm{S}$. The second transect at $\sim 42^{\circ} \mathrm{S}$ goes from the central gyre (Site U1368) through the southern gyre edge (Site U1370) to the northern edge of the upwelling region south of the gyre (Site U1371). The more northern suite of sites has been continuously located off-shore and beneath the low-productivity gyre water for many tens of millions of years (see the "Expedition 329 summary" chapter [Expedition 329 Scientists, 2011]). Equally, the southerly sites of the second transect have been located in the southern portion of the present-day gyre (Sites U1369 and U1370) or south of the gyre (Site U1371) during this time. Particularly at Site U1371, chlorophyll-a concentrations and primary productiv- 
ity are much higher than at all of the sites in the northern transect (see the "Expedition 329 summary" chapter [Expedition 329 Scientists, 2011]).

We recovered 208 sediment samples from the seven sites drilled (Sites U1365-U1371; Fig. F1) to analyze their particle size distribution. The primary objective of these analyses was to create a downcore profile of particle size at each site and to provide textural information that could be correlated with other types of data analyses made on adjacent samples. Samples were usually recovered at regular intervals, except for chert layers, which were avoided. Particle size distribution from this remote location can shed light on depositional conditions and diagenetic alterations and potentially provide information on eolian inputs and/or past deepwater circulation. Few studies have looked at particle size distribution in this area (see Rea, 1994, for a review).

\section{Methods}

The particle size data presented in this report were measured on a Micromeritics Sedigraph 5100 at the Paleoceanography laboratory of the School of Earth and Ocean Sciences at Cardiff University (United Kingdom). The Sedigraph technique is described in detail in Coakley and Syvitski (1991); further discussions of the operations of the instrument are provided by Stein (1985), Jones et al. (1988), and Bianchi et al. (1999). The Sedigraph method assumes that particles are dispersed in a fluid and settle according to Stoke's law. The Sedigraph determines the particle size distribution by the measurement of settling velocity and the mass fraction by relative absorption of low-energy X-ray. No specific sediment concentration is required for the analysis, providing the dispersed sample reduces the radiation beam by $40 \%-$ $60 \%$. This technique provides a rapid and accurate method (instrumental error $<1 \%$ ) for particle size analyses (e.g., Stein, 1985; Bianchi et al., 1999). The data are presented as a cumulative mass percent distribution in terms of equivalent spherical diameter. We used the Sedigraph because it is capable of sensing the total amount of material present and gives the whole size spectrum with satisfactory resolution $>1.5 \mu \mathrm{m}$.

Samples were collected and freeze-dried on board the JOIDES Resolution. Further sample preparation occurred at Cardiff University. Prior to particle size analysis, samples were disaggregated in $0.2 \%$ Calgon (sodium hexametaphosphate) dispersant on a rotating carousel for $24 \mathrm{~h}$. Coarse material $(>63 \mu \mathrm{m})$ was then removed by wet sieving over a $63 \mu \mathrm{m}$ mesh. The fine fraction was collected in a large $500 \mathrm{~mL}$ glass jar and left to settle before siphoning off the ex- cess water. Both the fine and coarse fractions were dried in an oven $\left(40^{\circ} \mathrm{C}\right)$ and weighed, allowing the weight percent in fine and coarse fractions to be calculated. Between 2 and $4 \mathrm{~g}$ of the fine fraction were weighed out for particle size analysis. The biogenic carbonate component was removed by slow digestion in $2 \mathrm{M}$ acetic acid solution for $24 \mathrm{~h}$ (twice for samples with $>1 \% \mathrm{CaCO}_{3}$ ). Biogenic silica was removed from Site U1371 samples by digestion using $6 \%(\mathrm{w} / \mathrm{w})$ sodium hydroxide at $80^{\circ} \mathrm{C}$ (water bath) for $5 \mathrm{~h}$. Samples were rinsed with deionized water, washed into a $60 \mathrm{~mL}$ Nalgene bottle with $0.2 \%$ Calgon solution, and placed on a rotating carousel overnight. All samples were sonicated for 1 min immediately before analysis on the Sedigraph.

Samples were run on the Sedigraph at a constant temperature of $35^{\circ} \mathrm{C}$ with an analysis range of $\sim 1.5-$ $63 \mu \mathrm{m}$, thus covering both the silt and clay range. Sedigraph samples were analyzed at the highest resolution possible, which includes an obligatory 11point smoothing specified by the manufacturer, providing a total of 269 data points (cumulative mass percentage finer). Output data files were analyzed using a custom-designed program (Sedilyze) that allows the full data to be both graphically and numerically displayed.

\section{Results}

We analyzed the particle size distribution of 208 sediment samples from the seven Expedition 329 drill sites. Particle size distributions are given in Tables T1, T2, T3, T4, T5, T6, and T7. We plot the downcore profile of mean grain size (e.g., graphic mean: $\left.\phi=\left(\phi_{16}+\phi_{50}+\phi_{84}\right) / 3\right)$ and sorting (e.g., standard deviation: $\left.\sigma_{\phi}=\left[\left(\phi_{84}-\phi_{16}\right) / 4\right]+\left[\left(\phi_{95}-\phi_{5}\right) / 6.6\right]\right)$, along with the summary of lithologic units and mineral composition for Sites U1365-U1371 in Figures F2, F3, F4, F5, F6, F7 and F8. In this study, we used the well-established size boundaries of $63 \mu \mathrm{m}$ separating sand and silt and $4 \mu \mathrm{m}$ separating silt and clay. The sediment collected during Expedition 329 is dominantly fine-grained clay and silt with negligible sand. Typical fine fraction contents are $>99 \mathrm{wt} \%$, with some samples having $>95 \mathrm{wt} \%$ of particles finer than $1.5 \mu \mathrm{m}$, in particular at Sites U1365 and U1370 (Tables T1, T6; Figs. F2, F7). Samples with $>75$ wt $\%$ of particles finer than $1.5 \mu \mathrm{m}$ were attributed an arbitrary mean particle size of $1.5 \mu \mathrm{m}(9.4 \phi)$ and no sorting. Site U1365 grains are mostly coarse clay with some very fine silt (Table T1). Sites U1366 and U1367 are mostly very fine silt (Tables T2, T3), as are the deeper parts of Sites U1368 and U1369 (Tables T4, T5). The upper part of Sites U1368 and U1369 consist of coarse clay. Site U1370 is mostly medium clay (Table 
T6). Site U1371 grains vary between coarse clay and very fine silt (Table T7). In most samples, the particle size distribution is negatively skewed to very negatively skewed and moderately sorted (poorly sorted at Site U1371; Table T7). However, a number of samples at the sites further offshore (Sites U1368 and U1369) are very well sorted (Tables T4, T5).

\section{Acknowledgments}

We thank the captain, crew, technicians, and science party aboard the R/V JOIDES Resolution for their assistance during Integrated Ocean Drilling Program (IODP) Expedition 329. All samples used in this study were collected during IODP Expedition 329. Natural Environment Research Council (NERC) funded participation of N. Dubois through grant NE/ I017895/1. Analyses and sample processing was funded through NERC grant NE/JO05282/1 and an ECORD Research Grant (2011) to N. Dubois. Data were reduced with the assistance of Chris Hein.

\section{References}

Bianchi, G.G., Hall, I.R., McCave, I.N., and Joseph, L., 1999. Measurement of the sortable silt current speed proxy using the Sedigraph 5100 and Coulter Multisizer IIe: precision and accuracy. Sedimentology, 46(6):10011014. doi:10.1046/j.1365-3091.1999.00256.x

Coakley, J.P., and Syvitski, J.P.M., 1991. SediGraph technique. In Syvitski, J.P.M. (Ed.), Principles, Methods, and
Application of Particle Size Analysis: Cambridge (Cambridge Univ. Press), 129-142. doi:10.1017/

CBO9780511626142.013

D'Hondt, S., Inagaki, F., Alvarez Zarikian, C.A., and the IODP Expedition 329 Science Party, 2013. IODP Expedition 329: life and habitability beneath the seafloor of the South Pacific Gyre. Sci. Drill., 15:4-10. doi:10.2204/ iodp.sd.15.01.2013

Expedition 329 Scientists, 2011. Expedition 329 summary. In D'Hondt, S., Inagaki, F., Alvarez Zarikian, C.A., and the Expedition 329 Scientists, Proc. IODP, 329: Tokyo (Integrated Ocean Drilling Program Management International, Inc.). doi:10.2204/iodp.proc.329.101.2011

Jones, K.P.N., McCave, I.N., and Patel, D., 1988. A computer-interfaced SediGraph for modal size analysis of fine-grained sediment. Sedimentology, 35(1):163-172. doi:10.1111/j.1365-3091.1988.tb00910.x

Rea, D.K., 1994. The paleoclimatic record provided by eolian deposition in the deep sea: the geologic history of wind. Rev. Geophys., 32(2):159-195. doi:10.1029/ 93RG03257

Stein, R., 1985. Rapid grain-size analyses of clay and silt fraction by Sedigraph 5000D: comparison with Coulter Counter and Atterberg methods. J. Sediment. Res., 55(4):590-593. http://archives.datapages.com/data/ sepm/journals/v55-58/data/055/055004/0590.htm

Initial receipt: 14 August 2013

Acceptance: 6 February 2014

Publication: 9 APRIL 2014

MS 329-201 
Figure F1. Map of annual chlorophyll-a concentrations overlain by Expedition 329 site locations (white circles). White lines indicate basement age in 10 m.y. increments. Black lines indicate site positions over geologic time (by D’Hondt et al., 2013).

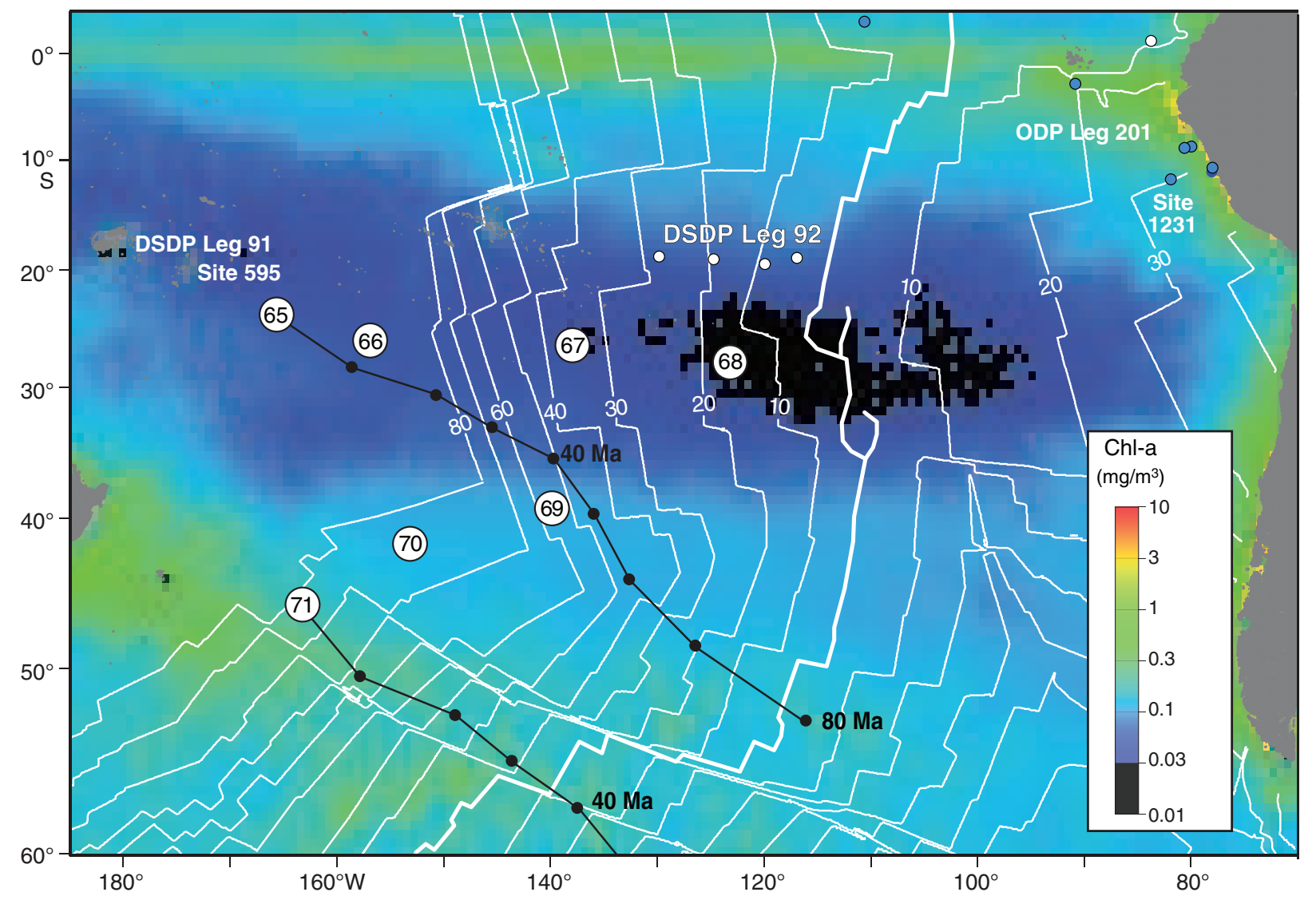


Figure F2. Downcore distribution of mean grain size $(\phi)$ and sorting $\left(\sigma_{\phi}\right)$, along with the lithostratigraphic units and mineral composition of Site U1365 (modified from Expedition 329 Scientists, 2011). RSO = red-brown to yellow-brown semiopaque oxide.

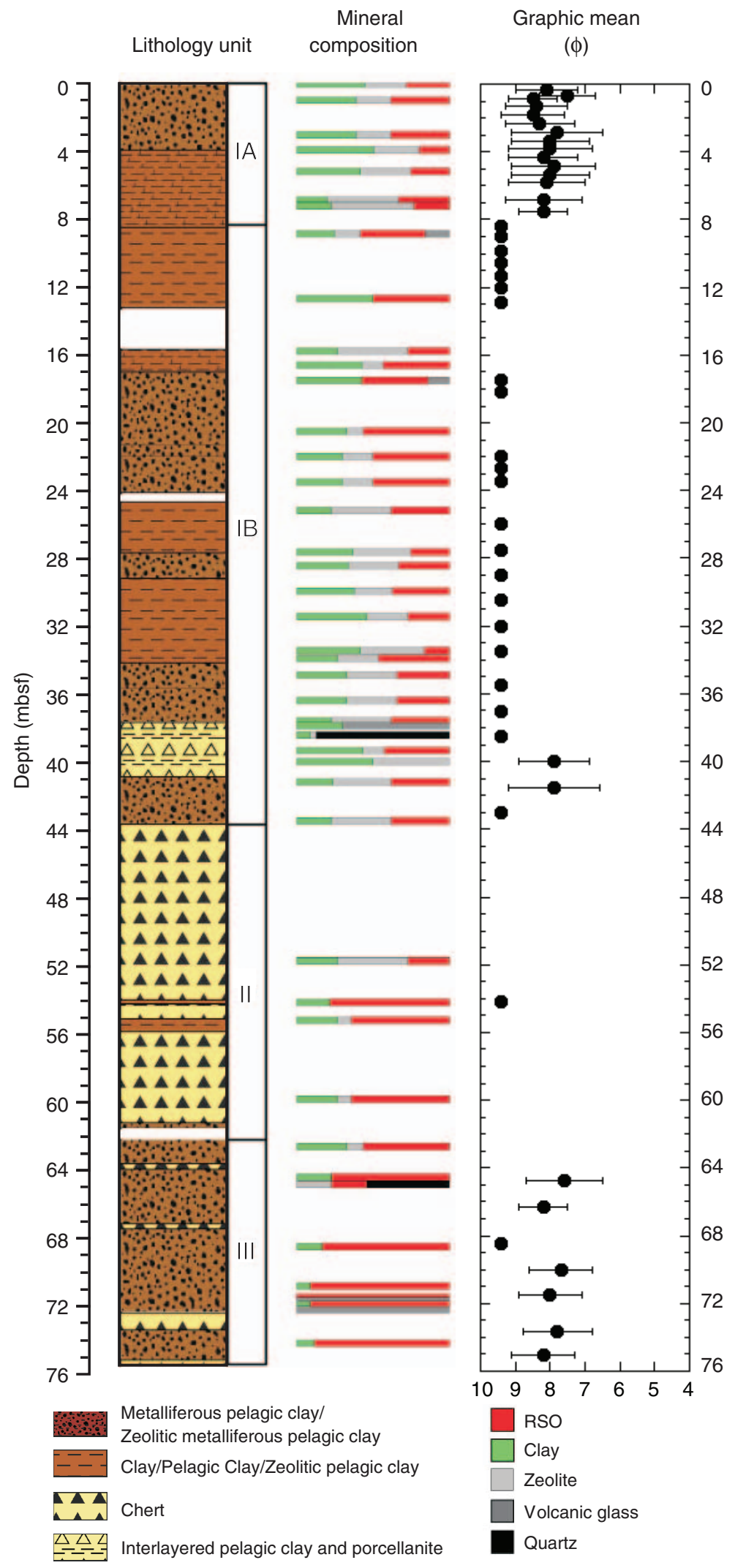


Figure F3. Downcore distribution of mean grain size $(\phi)$ and sorting $\left(\sigma_{\phi}\right)$, along with the lithostratigraphic units and mineral composition of Site U1366 (modified from Expedition 329 Scientists, 2011). RSO = red-brown to yellow-brown semiopaque oxide.

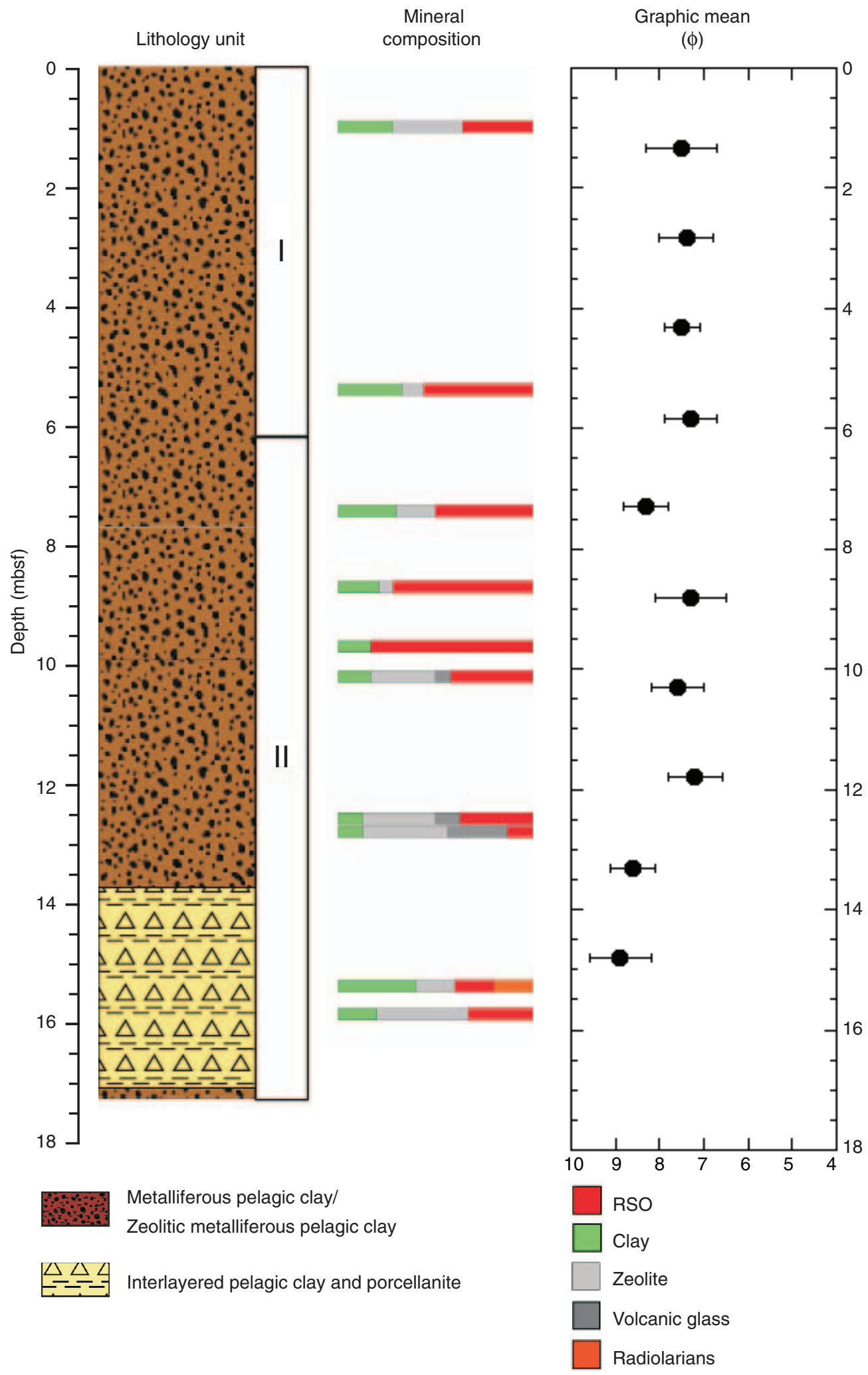


Figure F4. Downcore distribution of mean grain size $(\phi)$ and sorting $\left(\sigma_{\phi}\right)$, along with the lithostratigraphic units and mineral composition of Site U1367 (modified from Expedition 329 Scientists, 2011). RSO = red-brown to yellow-brown semiopaque oxide.

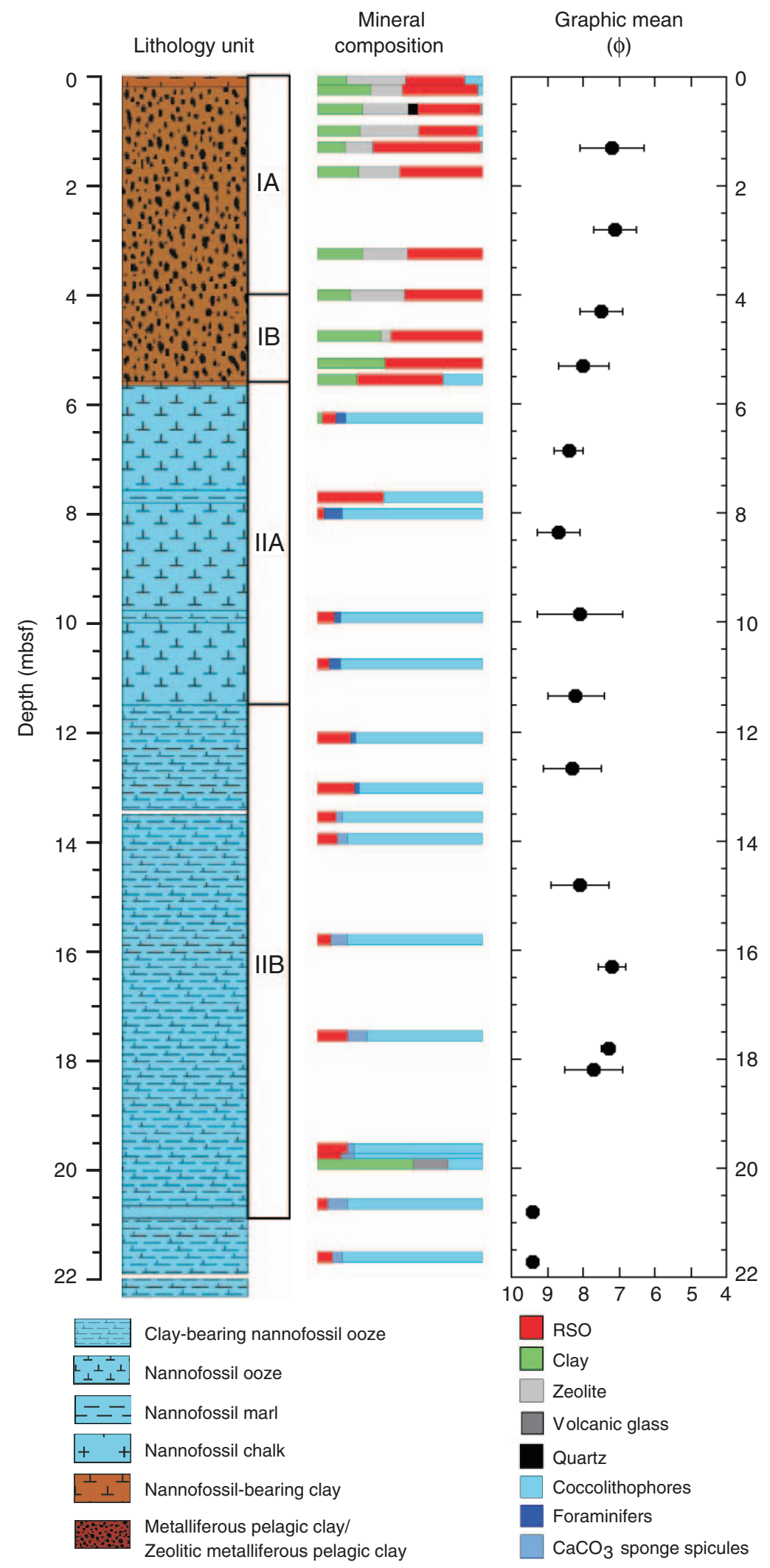


Figure F5. Downcore distribution of mean grain size $(\phi)$ and sorting $\left(\sigma_{\phi}\right)$, along with the lithostratigraphic units and mineral composition of Site U1368 (modified from Expedition 329 Scientists, 2011). RSO = red-brown to yellow-brown semiopaque oxide.

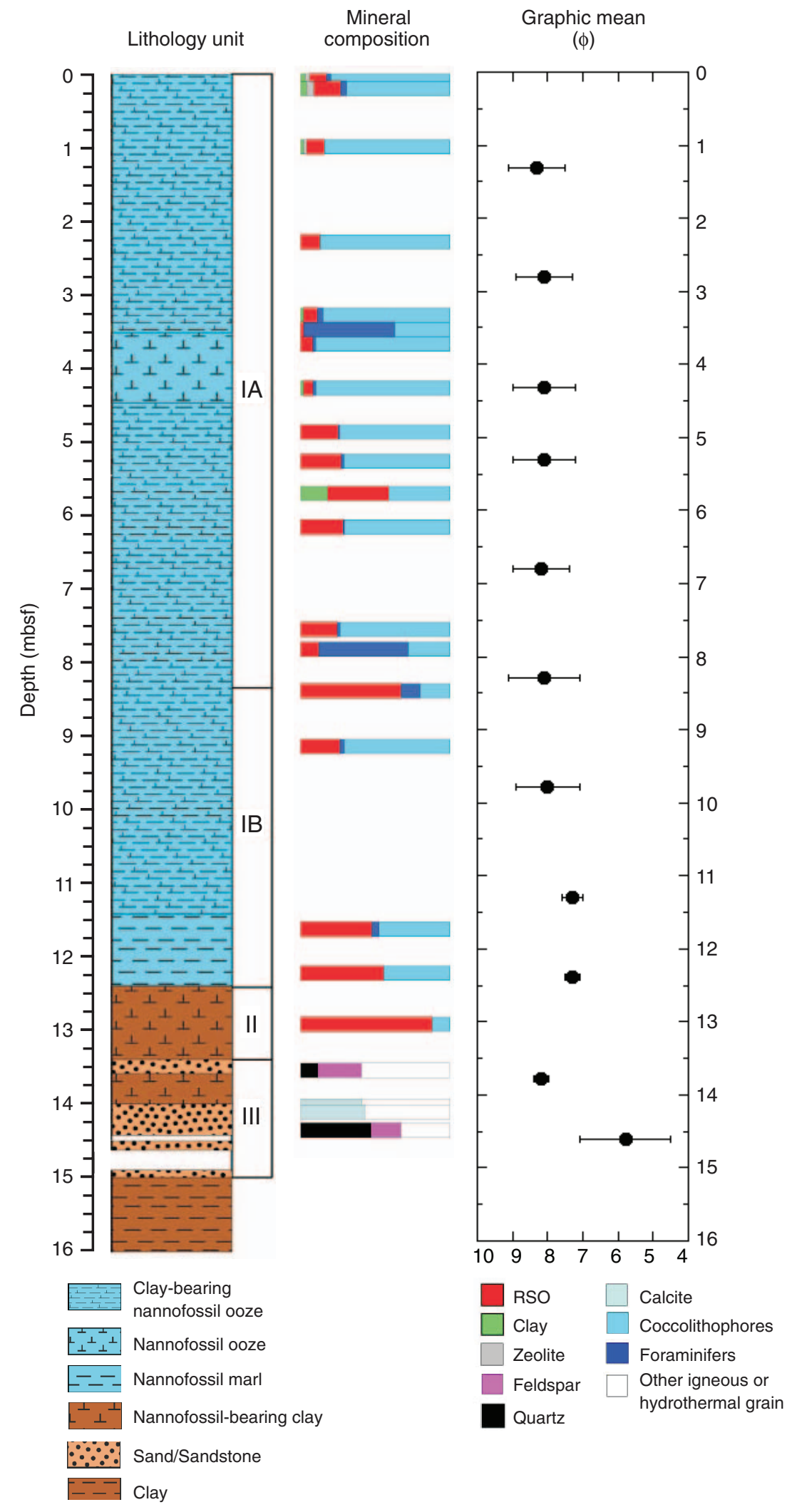


Figure F6. Downcore distribution of mean grain size $(\phi)$ and sorting $\left(\sigma_{\phi}\right)$, along with the lithostratigraphic units and mineral composition of Site U1369 (modified from Expedition 329 Scientists, 2011). RSO = red-brown to yellow-brown semiopaque oxide.

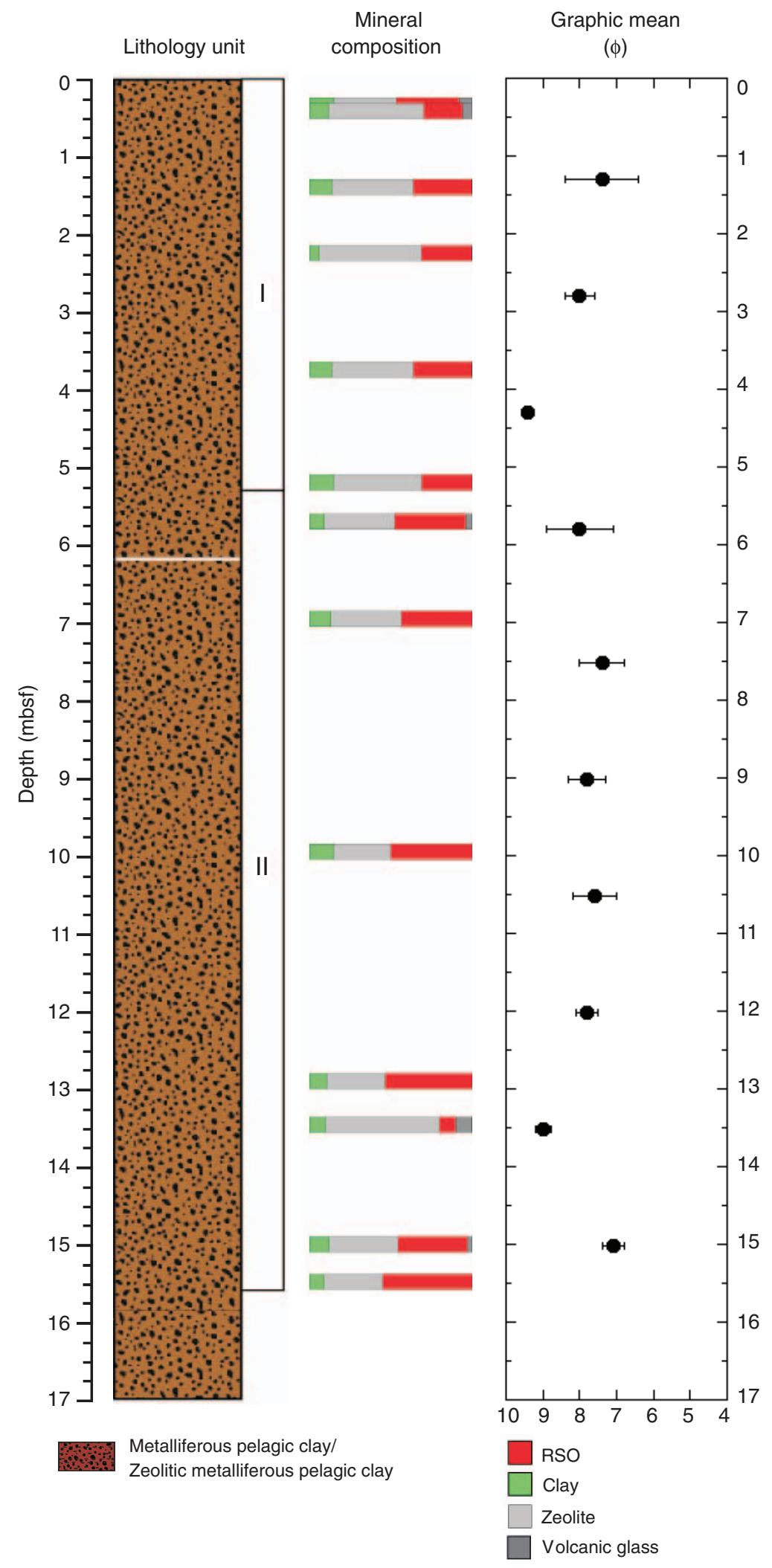


Figure F7. Downcore distribution of mean grain size $(\phi)$ and sorting $\left(\sigma_{\phi}\right)$, along with the lithostratigraphic units and mineral composition of Site U1370 (modified from Expedition 329 Scientists, 2011). RSO = red-brown to yellow-brown semiopaque oxide.

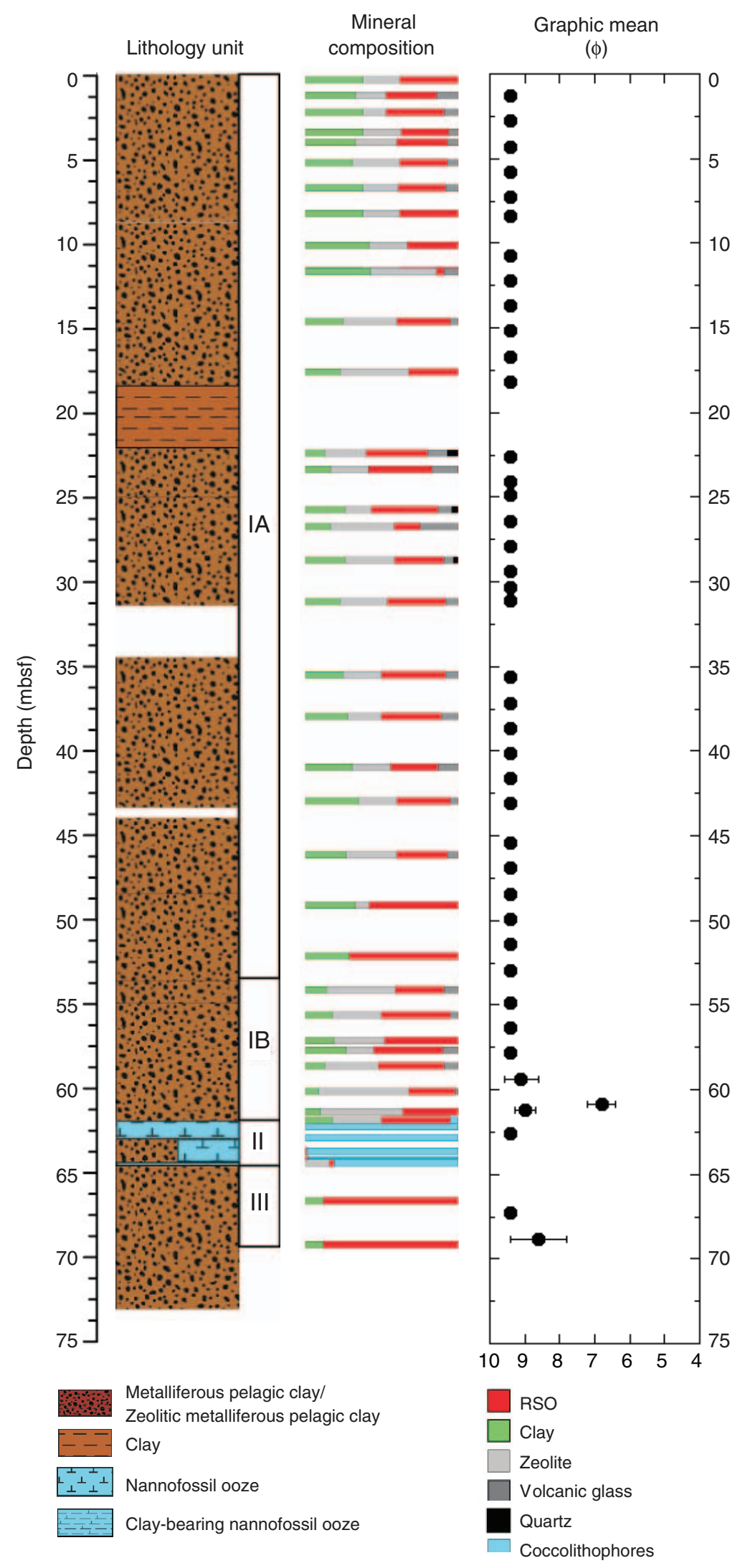


Figure F8. Downcore distribution of mean grain size $(\phi)$ and sorting $\left(\sigma_{\phi}\right)$, along with the lithostratigraphic units and mineral composition of Site U1371 (modified from Expedition 329 Scientists, 2011). RSO = red-brown to yellow-brown semiopaque oxide.

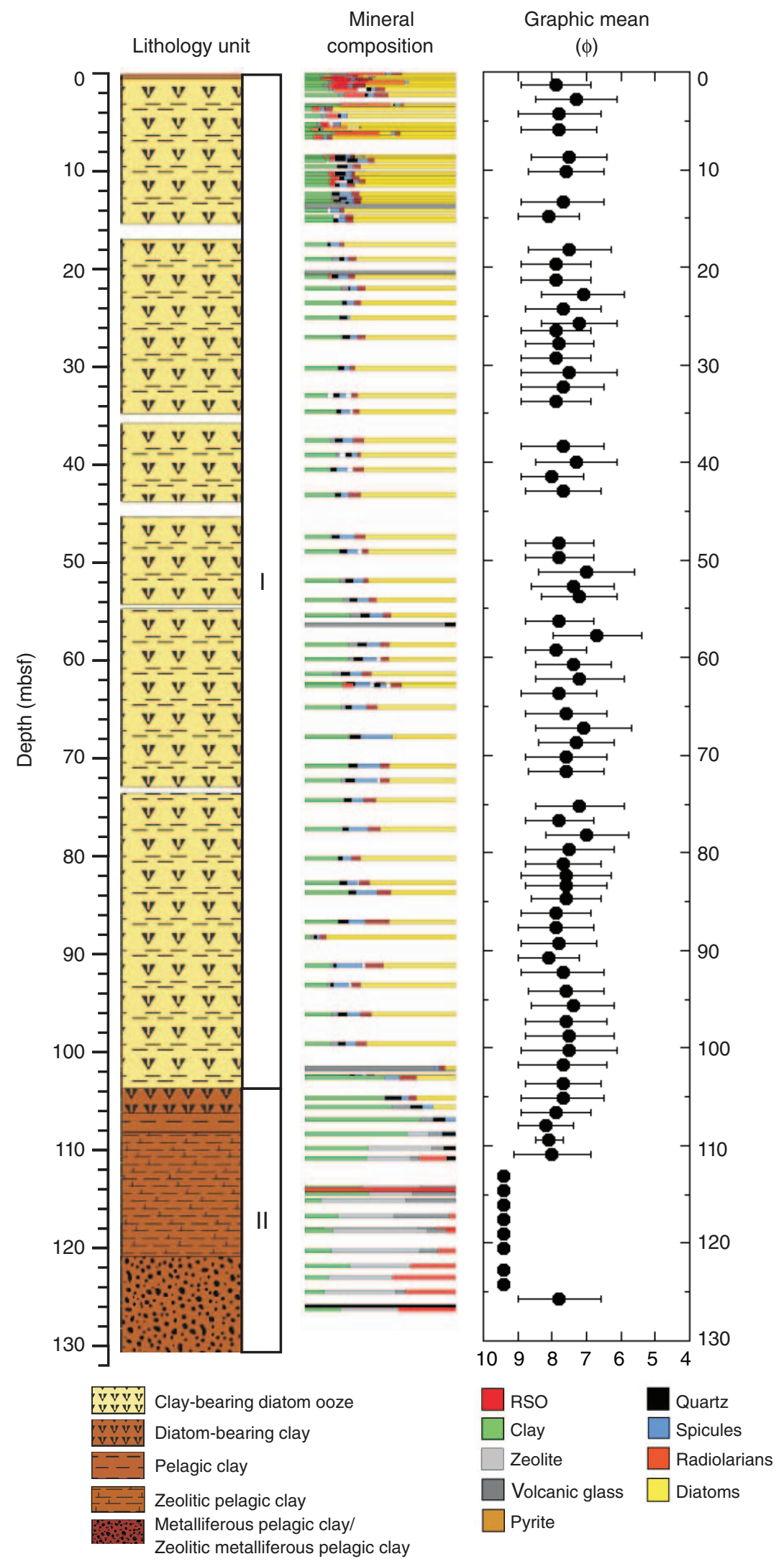


Table T1. Particle size analysis results, Site U1365.

\begin{tabular}{|c|c|c|c|c|c|c|c|c|c|}
\hline \multirow{2}{*}{$\begin{array}{l}\text { Core, section, } \\
\text { interval }(\mathrm{cm})\end{array}$} & \multirow{2}{*}{$\begin{array}{l}\text { Depth } \\
\text { (mbsf) }\end{array}$} & \multicolumn{3}{|c|}{ Particle size (wt\%) } & \multirow{2}{*}{$\begin{array}{c}\text { Mean } \\
(\mu \mathrm{m})\end{array}$} & \multirow{2}{*}{$\begin{array}{c}\text { Mean } \\
(\phi)\end{array}$} & \multirow{2}{*}{$\begin{array}{l}\text { Sorting } \\
(\phi)\end{array}$} & \multirow{2}{*}{$\begin{array}{c}\text { Grain size } \\
\text { (Wentworth) }\end{array}$} & \multirow[b]{2}{*}{ Sorting } \\
\hline & & Sand & Silt & Clay & & & & & \\
\hline \multicolumn{10}{|l|}{ 329-U1365A- } \\
\hline $1 \mathrm{H}-1,32-36$ & 0.34 & 0.00 & 46.51 & 53.49 & 3.68 & 8.09 & 0.95 & Coarse clay & Moderately sorted \\
\hline $1 \mathrm{H}-1,65-69$ & 0.67 & 0.00 & 77.72 & 22.28 & 5.42 & 7.53 & 0.75 & Very fine silt & Moderately sorted \\
\hline $1 \mathrm{H}-1,82-86$ & 0.84 & 0.15 & 22.49 & 77.36 & 2.78 & 8.49 & 0.70 & Coarse clay & Moderately sorted \\
\hline $1 \mathrm{H}-1,132-136$ & 1.34 & 0.00 & 28.19 & 71.81 & 3.01 & 8.38 & 0.88 & Coarse clay & Moderately sorted \\
\hline $1 \mathrm{H}-2,32-36$ & 1.84 & 0.15 & 20.75 & 79.10 & 2.76 & 8.50 & 0.86 & Coarse clay & Moderately sorted \\
\hline $1 \mathrm{H}-2,82-86$ & 2.34 & 0.00 & 30.63 & 69.37 & 3.14 & 8.31 & 0.99 & Coarse clay & Moderately sorted \\
\hline $1 \mathrm{H}-2,132-136$ & 2.84 & 0.26 & 43.33 & 56.41 & 4.52 & 7.79 & 1.30 & Coarse clay & Poorly sorted \\
\hline $1 \mathrm{H}-3,32-36$ & 3.34 & 0.03 & 38.29 & 61.68 & 3.82 & 8.03 & 1.14 & Coarse clay & Poorly sorted \\
\hline $1 \mathrm{H}-3,82-86$ & 3.84 & 0.09 & 38.35 & 61.55 & 4.03 & 7.95 & 1.25 & Coarse clay & Poorly sorted \\
\hline $1 \mathrm{H}-3,132-136$ & 4.34 & 0.00 & 33.94 & 66.06 & 3.42 & 8.19 & 1.00 & Coarse clay & Moderately sorted \\
\hline $1 \mathrm{H}-4,32-36$ & 4.84 & 0.00 & 41.46 & 58.54 & 4.26 & 7.88 & 1.24 & Coarse clay & Poorly sorted \\
\hline $1 \mathrm{H}-4,82-86$ & 5.34 & 0.10 & 37.46 & 62.44 & 3.90 & 8.00 & 1.15 & Coarse clay & Poorly sorted \\
\hline $1 \mathrm{H}-4,132-136$ & 5.84 & 0.20 & 36.98 & 62.81 & 3.58 & 8.13 & 1.09 & Coarse clay & Poorly sorted \\
\hline $2 \mathrm{H}-1,65-69$ & 6.87 & 0.00 & 33.76 & 66.24 & 3.32 & 8.23 & 1.10 & Coarse clay & Poorly sorted \\
\hline $2 \mathrm{H}-1,130-134$ & 7.52 & 0.01 & 31.05 & 68.94 & 3.35 & 8.22 & 0.66 & Coarse clay & Moderately well sorted \\
\hline $2 \mathrm{H}-2,65-69$ & 8.37 & ND & ND & ND & 1.50 & 9.40 & ND & Medium-fine clay & ND \\
\hline $2 \mathrm{H}-2,130-134$ & 9.02 & ND & ND & ND & 1.50 & 9.40 & ND & Medium-fine clay & ND \\
\hline $2 \mathrm{H}-3,65-69$ & 9.87 & ND & ND & ND & 1.50 & 9.40 & ND & Medium-fine clay & ND \\
\hline $2 \mathrm{H}-3,130-134$ & 10.52 & ND & ND & ND & 1.50 & 9.40 & ND & Medium-fine clay & ND \\
\hline $2 \mathrm{H}-4,65-69$ & 11.37 & ND & ND & ND & 1.50 & 9.40 & ND & Medium-fine clay & ND \\
\hline $2 \mathrm{H}-4,130-134$ & 12.02 & ND & ND & ND & 1.50 & 9.40 & ND & Medium-fine clay & ND \\
\hline $2 \mathrm{H}-5,65-69$ & 12.87 & ND & ND & ND & 1.50 & 9.40 & ND & Medium-fine clay & ND \\
\hline $3 \mathrm{H}-2,62-66$ & 17.46 & ND & ND & ND & 1.50 & 9.40 & ND & Medium-fine clay & ND \\
\hline $3 \mathrm{H}-2,136-140$ & 18.20 & ND & ND & ND & 1.50 & 9.40 & ND & Medium-fine clay & ND \\
\hline $3 \mathrm{H}-5,66-70$ & 22.00 & ND & ND & ND & 1.50 & 9.40 & ND & Medium-fine clay & ND \\
\hline $3 \mathrm{H}-5,133-137$ & 22.67 & ND & ND & ND & 1.50 & 9.40 & ND & Medium-fine clay & ND \\
\hline $3 H-6,59-63$ & 23.43 & ND & ND & ND & 1.50 & 9.40 & ND & Medium-fine clay & ND \\
\hline $4 \mathrm{H}-1,130-132$ & 26.01 & ND & ND & ND & 1.50 & 9.40 & ND & Medium-fine clay & ND \\
\hline $4 \mathrm{H}-2,133-135$ & 27.54 & ND & ND & ND & 1.50 & 9.40 & ND & Medium-fine clay & ND \\
\hline $4 \mathrm{H}-3,130-132$ & 29.01 & ND & ND & ND & 1.50 & 9.40 & ND & Medium-fine clay & ND \\
\hline $4 \mathrm{H}-4,130-132$ & 30.51 & ND & ND & ND & 1.50 & 9.40 & ND & Medium-fine clay & ND \\
\hline $4 \mathrm{H}-5,130-132$ & 32.01 & ND & ND & ND & 1.50 & 9.40 & ND & Medium-fine clay & ND \\
\hline $4 \mathrm{H}-6,130-132$ & 33.51 & ND & ND & ND & 1.50 & 9.40 & ND & Medium-fine clay & ND \\
\hline $5 \mathrm{H}-1,132-134$ & 35.53 & ND & ND & ND & 1.50 & 9.40 & ND & Medium-fine clay & ND \\
\hline $5 \mathrm{H}-2,132-134$ & 37.03 & ND & ND & ND & 1.50 & 9.40 & ND & Medium-fine clay & ND \\
\hline $5 \mathrm{H}-3,132-134$ & 38.53 & ND & ND & ND & 1.50 & 9.40 & ND & Medium-fine clay & ND \\
\hline $5 \mathrm{H}-4,131-133$ & 40.02 & 0.27 & 48.47 & 51.27 & 4.14 & 7.92 & 1.04 & Coarse clay & Poorly sorted \\
\hline $5 \mathrm{H}-5,131-133$ & 41.52 & 0.00 & 43.69 & 56.31 & 4.11 & 7.93 & 1.26 & Coarse clay & Poorly sorted \\
\hline $5 \mathrm{H}-6,132-134$ & 43.03 & ND & ND & ND & 1.50 & 9.40 & ND & Medium-fine clay & ND \\
\hline $14 \mathrm{H}-1,136-138$ & 54.17 & ND & ND & ND & 1.50 & 9.40 & ND & Medium-fine clay & ND \\
\hline $23 \mathrm{H}-1,148-150$ & 64.79 & 0.05 & 58.48 & 41.47 & 5.13 & 7.61 & 1.12 & Coarse clay & Poorly sorted \\
\hline $23 \mathrm{H}-2,148-150$ & 66.29 & 0.00 & 34.61 & 65.39 & 3.34 & 8.23 & 0.72 & Coarse clay & Moderately sorted \\
\hline $24 \mathrm{H}-1,130-132$ & 68.51 & ND & ND & ND & 1.50 & 9.40 & ND & Medium-fine clay & ND \\
\hline $24 \mathrm{H}-2,130-132$ & 70.03 & 0.00 & 62.19 & 37.81 & 4.85 & 7.69 & 0.93 & Very fine silt & Moderately sorted \\
\hline $24 \mathrm{H}-3,130-132$ & 71.53 & 0.00 & 45.22 & 54.78 & 3.87 & 8.01 & 0.90 & Coarse clay & Moderately sorted \\
\hline $25 \mathrm{H}-1,122-124$ & 73.63 & 0.00 & 55.48 & 44.52 & 4.55 & 7.78 & 1.00 & Coarse clay & Moderately sorted \\
\hline $25 \mathrm{H}-2,115-119$ & 75.06 & 0.01 & 38.79 & 61.20 & 3.33 & 8.23 & 0.86 & Coarse clay & Moderately sorted \\
\hline
\end{tabular}

$\mathrm{ND}=$ not detected 
Table T2. Particle size analysis results, Site U1366.

\begin{tabular}{|c|c|c|c|c|c|c|c|c|c|}
\hline \multirow{2}{*}{$\begin{array}{l}\text { Core, section, } \\
\text { interval }(\mathrm{cm})\end{array}$} & \multirow{2}{*}{$\begin{array}{l}\text { Depth } \\
\text { (mbsf) }\end{array}$} & \multicolumn{3}{|c|}{ Particle size (wt\%) } & \multirow{2}{*}{$\begin{array}{c}\text { Mean } \\
(\mu \mathrm{m})\end{array}$} & \multirow{2}{*}{$\begin{array}{c}\text { Mean } \\
(\phi)\end{array}$} & \multirow{2}{*}{$\begin{array}{l}\text { Sorting } \\
(\phi)\end{array}$} & \multirow{2}{*}{$\begin{array}{c}\text { Grain size } \\
\text { (Wentworth) }\end{array}$} & \multirow[b]{2}{*}{ Sorting } \\
\hline & & Sand & Silt & Clay & & & & & \\
\hline \multicolumn{10}{|l|}{ 329-U1366C- } \\
\hline $1 \mathrm{H}-1,132-134$ & 1.33 & 0.02 & 84.15 & 15.83 & 5.81 & 7.43 & 0.72 & Very fine silt & Moderately sorted \\
\hline $1 \mathrm{H}-2,132-134$ & 2.83 & 0.00 & 91.27 & 8.73 & 5.88 & 7.41 & 0.59 & Very fine silt & Moderately well sorted \\
\hline $1 \mathrm{H}-3,132-134$ & 4.33 & 0.14 & 95.20 & 4.66 & 5.50 & 7.51 & 0.44 & Very fine silt & Well sorted \\
\hline $1 \mathrm{H}-4,132-134$ & 5.83 & 0.00 & 95.35 & 4.65 & 6.63 & 7.24 & 0.50 & Very fine silt & Moderately well sorted \\
\hline $2 \mathrm{H}-1,130-132$ & 7.31 & 0.00 & 23.02 & 76.98 & 3.19 & 8.29 & 0.47 & Coarse clay & Well sorted \\
\hline $2 \mathrm{H}-2,130-132$ & 8.81 & 0.00 & 88.64 & 11.36 & 6.54 & 7.26 & 0.78 & Very fine silt & Moderately sorted \\
\hline $2 \mathrm{H}-3,130-132$ & 10.31 & 0.06 & 82.72 & 17.22 & 5.18 & 7.59 & 0.57 & Very fine silt & Moderately well sorted \\
\hline $2 \mathrm{H}-4,130-132$ & 11.81 & 0.00 & 98.50 & 1.50 & 6.93 & 7.17 & 0.64 & Very fine silt & Moderately well sorted \\
\hline $2 \mathrm{H}-5,130-132$ & 13.31 & 0.02 & 8.59 & 91.39 & 2.09 & 8.90 & 0.45 & Medium clay & Well sorted \\
\hline $2 \mathrm{H}-6,130-132$ & 14.81 & 0.00 & 16.69 & 83.31 & 2.38 & 8.72 & 0.71 & Medium clay & Moderately sorted \\
\hline
\end{tabular}

Table T3. Particle size analysis results, Site U1367.

\begin{tabular}{|c|c|c|c|c|c|c|c|c|c|}
\hline \multirow{2}{*}{$\begin{array}{l}\text { Core, section, } \\
\text { interval }(\mathrm{cm})\end{array}$} & \multirow{2}{*}{$\begin{array}{l}\text { Depth } \\
\text { (mbsf) }\end{array}$} & \multicolumn{3}{|c|}{ Particle size (wt\%) } & \multirow{2}{*}{$\begin{array}{c}\text { Mean } \\
(\mu \mathrm{m})\end{array}$} & \multirow{2}{*}{$\begin{array}{c}\text { Mean } \\
(\phi)\end{array}$} & \multirow{2}{*}{$\begin{array}{c}\text { Sorting } \\
(\phi)\end{array}$} & \multirow{2}{*}{$\begin{array}{c}\text { Grain size } \\
\text { (Wentworth) }\end{array}$} & \multirow[b]{2}{*}{ Sorting } \\
\hline & & Sand & Silt & Clay & & & & & \\
\hline \multicolumn{10}{|l|}{ 329-U1367B- } \\
\hline $1 \mathrm{H}-1,130-132$ & 1.31 & 0.00 & 85.72 & 14.28 & 6.70 & 7.22 & 0.88 & Very fine silt & Moderately sorted \\
\hline $1 \mathrm{H}-2,130-132$ & 2.81 & 0.01 & 94.13 & 5.86 & 7.10 & 7.14 & 0.64 & Very fine silt & Moderately well sorted \\
\hline $1 \mathrm{H}-3,130-132$ & 4.31 & 0.02 & 81.88 & 18.10 & 5.39 & 7.54 & 0.65 & Very fine silt & Moderately well sorted \\
\hline $1 \mathrm{H}-4,80-82$ & 5.31 & 0.01 & 59.37 & 40.61 & 3.99 & 7.97 & 0.72 & Very fine silt & Moderately sorted \\
\hline $2 \mathrm{H}-1,135-137$ & 6.86 & 0.00 & 13.55 & 86.45 & 2.94 & 8.41 & 0.38 & Coarse clay & Well sorted \\
\hline $2 \mathrm{H}-2,135-137$ & 8.36 & 0.00 & 14.22 & 85.78 & 2.39 & 8.71 & 0.55 & Coarse clay & Moderately well sorted \\
\hline $2 \mathrm{H}-3,135-137$ & 9.86 & 0.37 & 43.97 & 55.66 & 3.62 & 8.11 & 1.15 & Coarse clay & Poorly sorted \\
\hline $2 \mathrm{H}-4,135-137$ & 11.36 & 0.00 & 37.28 & 62.72 & 3.31 & 8.24 & 0.78 & Coarse clay & Moderately sorted \\
\hline $2 \mathrm{H}-5,116-118$ & 12.67 & 0.00 & 33.07 & 66.93 & 3.15 & 8.31 & 0.77 & Coarse clay & Moderately sorted \\
\hline $3 \mathrm{H}-1,130-132$ & 14.81 & 0.00 & 42.55 & 57.45 & 3.55 & 8.14 & 0.76 & Coarse clay & Moderately sorted \\
\hline $3 \mathrm{H}-2,130-132$ & 16.31 & 0.00 & 94.91 & 5.09 & 6.85 & 7.19 & 0.39 & Very fine silt & Well sorted \\
\hline $3 \mathrm{H}-3,130-132$ & 17.81 & 0.01 & 99.74 & 0.25 & 6.15 & 7.34 & 0.20 & Very fine silt & Very well sorted \\
\hline $3 \mathrm{H}-4,20-22$ & 18.21 & 0.02 & 76.38 & 23.60 & 4.81 & 7.70 & 0.76 & Very fine silt & Moderately sorted \\
\hline $3 \mathrm{H}-5,130-132$ & 20.81 & ND & ND & ND & 1.50 & 9.40 & ND & Medium-fine clay & ND \\
\hline $3 \mathrm{H}-6,72-74$ & 21.73 & ND & ND & ND & 1.50 & 9.40 & ND & Medium-fine clay & ND \\
\hline
\end{tabular}

$\mathrm{ND}=$ not detected

Table T4. Particle size analysis results, Site U1368.

\begin{tabular}{|c|c|c|c|c|c|c|c|c|c|}
\hline \multirow{2}{*}{$\begin{array}{l}\text { Core, section, } \\
\text { interval }(\mathrm{cm})\end{array}$} & \multirow{2}{*}{$\begin{array}{l}\text { Depth } \\
\text { (mbsf) }\end{array}$} & \multicolumn{3}{|c|}{ Particle size (wt\%) } & \multirow{2}{*}{$\begin{array}{l}\text { Mean } \\
(\mu \mathrm{m})\end{array}$} & \multirow{2}{*}{$\begin{array}{c}\text { Mean } \\
(\phi)\end{array}$} & \multirow{2}{*}{$\begin{array}{l}\text { Sorting } \\
(\phi)\end{array}$} & \multirow{2}{*}{$\begin{array}{c}\text { Grain size } \\
\text { (Wentworth) }\end{array}$} & \multirow[b]{2}{*}{ Sorting } \\
\hline & & Sand & Silt & Clay & & & & & \\
\hline \multicolumn{10}{|l|}{ 329-U1368B- } \\
\hline $1 \mathrm{H}-1,130-132$ & 1.31 & 0.00 & 35.50 & 64.50 & 3.16 & 8.30 & 0.79 & Coarse clay & Moderately sorted \\
\hline $1 \mathrm{H}-2,130-132$ & 2.81 & 0.03 & 42.95 & 57.02 & 3.56 & 8.13 & 0.82 & Coarse clay & Moderately sorted \\
\hline $1 \mathrm{H}-3,130-132$ & 4.31 & 0.00 & 43.38 & 56.62 & 3.65 & 8.10 & 0.87 & Coarse clay & Moderately sorted \\
\hline $1 \mathrm{H}-4,80-82$ & 5.31 & 0.00 & 42.54 & 57.46 & 3.61 & 8.11 & 0.86 & Coarse clay & Moderately sorted \\
\hline $2 \mathrm{H}-1,128-130$ & 6.79 & 0.01 & 42.18 & 57.81 & 3.49 & 8.16 & 0.79 & Coarse clay & Moderately sorted \\
\hline $2 \mathrm{H}-2,128-130$ & 8.29 & 0.20 & 43.86 & 55.94 & 3.71 & 8.07 & 1.01 & Coarse clay & Poorly sorted \\
\hline $2 \mathrm{H}-3,128-130$ & 9.79 & 0.06 & 49.81 & 50.13 & 3.91 & 8.00 & 0.87 & Coarse clay & Moderately sorted \\
\hline $2 \mathrm{H}-4,128-130$ & 11.29 & 0.00 & 98.15 & 1.85 & 6.24 & 7.32 & 0.27 & Very fine silt & Very well sorted \\
\hline $2 \mathrm{H}-5,88-90$ & 12.39 & 0.01 & 99.85 & 0.14 & 6.24 & 7.32 & 0.20 & Very fine silt & Very well sorted \\
\hline $2 \mathrm{H}-6,128-130$ & 13.79 & 0.00 & 11.24 & 88.76 & 3.28 & 8.25 & 0.21 & Coarse clay & Very well sorted \\
\hline $2 \mathrm{H}-\mathrm{CC}, 10-12$ & 14.61 & 0.44 & 89.64 & 9.92 & 17.71 & 5.82 & 1.35 & Medium silt & Poorly sorted \\
\hline
\end{tabular}


Table T5. Particle size analysis results, Site U1369.

\begin{tabular}{|c|c|c|c|c|c|c|c|c|c|}
\hline \multirow{2}{*}{$\begin{array}{l}\text { Core, section, } \\
\text { interval }(\mathrm{cm})\end{array}$} & \multirow{2}{*}{$\begin{array}{l}\text { Depth } \\
\text { (mbsf) }\end{array}$} & \multicolumn{3}{|c|}{ Particle size (wt\%) } & \multirow{2}{*}{$\begin{array}{c}\text { Mean } \\
(\mu \mathrm{m})\end{array}$} & \multirow{2}{*}{$\begin{array}{c}\text { Mean } \\
(\phi)\end{array}$} & \multirow{2}{*}{$\begin{array}{l}\text { Sorting } \\
(\phi)\end{array}$} & \multirow{2}{*}{$\begin{array}{l}\text { Grain size } \\
\text { (Wentworth) }\end{array}$} & \multirow[b]{2}{*}{ Sorting } \\
\hline & & Sand & Silt & Clay & & & & & \\
\hline \multicolumn{10}{|l|}{ 329-U1369B- } \\
\hline $1 \mathrm{H}-1,130-132$ & 1.31 & 0.00 & 69.21 & 30.79 & 6.02 & 7.38 & 0.97 & Very fine silt & Moderately sorted \\
\hline $1 \mathrm{H}-2,130-132$ & 2.81 & 0.00 & 39.12 & 60.88 & 3.85 & 8.02 & 0.35 & Coarse clay & Well sorted \\
\hline $1 \mathrm{H}-3,130-132$ & 4.31 & ND & ND & ND & 1.50 & 9.40 & ND & Medium-fine clay & ND \\
\hline $1 \mathrm{H}-4,130-132$ & 5.81 & 0.00 & 43.81 & 56.19 & 3.84 & 8.03 & 0.93 & Coarse clay & Moderately sorted \\
\hline $2 \mathrm{H}-1,130-132$ & 7.51 & 0.02 & 90.41 & 9.57 & 5.77 & 7.44 & 0.64 & Very fine silt & Moderately well sorted \\
\hline $2 \mathrm{H}-2,130-132$ & 9.01 & 0.06 & 57.24 & 42.70 & 4.39 & 7.83 & 0.54 & Very fine silt & Moderately well sorted \\
\hline $2 \mathrm{H}-3,130-132$ & 10.51 & 0.02 & 81.70 & 18.28 & 5.13 & 7.61 & 0.58 & Very fine silt & Moderately well sorted \\
\hline $2 \mathrm{H}-4,130-132$ & 12.01 & 0.00 & 82.92 & 17.08 & 4.64 & 7.75 & 0.33 & Very fine silt & Very well sorted \\
\hline $2 \mathrm{H}-5,130-132$ & 13.51 & 0.00 & 3.03 & 96.97 & 2.01 & 8.96 & 0.20 & Coarse clay & Very well sorted \\
\hline $2 \mathrm{H}-6,130-132$ & 15.01 & 0.00 & 98.17 & 1.83 & 7.06 & 7.15 & 0.31 & Very fine silt & Very well sorted \\
\hline
\end{tabular}

$\mathrm{ND}=$ not detected

Table T6. Particle size analysis results, Site U1370.

\begin{tabular}{|c|c|c|c|c|c|c|c|c|c|}
\hline \multirow{2}{*}{$\begin{array}{l}\text { Core, section, } \\
\text { interval }(\mathrm{cm})\end{array}$} & \multirow{2}{*}{$\begin{array}{l}\text { Depth } \\
\text { (mbsf) }\end{array}$} & \multicolumn{3}{|c|}{ Particle size (wt\%) } & \multirow{2}{*}{$\begin{array}{l}\text { Mean } \\
(\mu \mathrm{m})\end{array}$} & \multirow{2}{*}{$\begin{array}{c}\text { Mean } \\
(\phi)\end{array}$} & \multirow{2}{*}{$\begin{array}{l}\text { Sorting } \\
(\phi)\end{array}$} & \multirow{2}{*}{$\begin{array}{c}\text { Grain size } \\
\text { (Wentworth) }\end{array}$} & \multirow[b]{2}{*}{ Sorting } \\
\hline & & Sand & Silt & Clay & & & & & \\
\hline \multicolumn{10}{|l|}{ 329-U1370D- } \\
\hline $1 \mathrm{H}-1,130-132$ & 1.31 & ND & ND & ND & 1.50 & 9.40 & ND & Medium-fine clay & ND \\
\hline $1 \mathrm{H}-2,130-132$ & 2.81 & ND & ND & ND & 1.50 & 9.40 & ND & Medium-fine clay & ND \\
\hline $1 \mathrm{H}-3,130-132$ & 4.31 & ND & ND & ND & 1.50 & 9.40 & ND & Medium-fine clay & ND \\
\hline $1 \mathrm{H}-4,130-132$ & 5.81 & ND & ND & ND & 1.50 & 9.40 & ND & Medium-fine clay & ND \\
\hline $1 \mathrm{H}-5,130-132$ & 7.31 & ND & ND & ND & 1.50 & 9.40 & ND & Medium-fine clay & ND \\
\hline $1 \mathrm{H}-6,90-92$ & 8.41 & ND & ND & ND & 1.50 & 9.40 & ND & Medium-fine clay & ND \\
\hline $2 \mathrm{H}-2,130-132$ & 10.71 & ND & ND & ND & 1.50 & 9.40 & ND & Medium-fine clay & ND \\
\hline $2 \mathrm{H}-3,130-132$ & 12.21 & ND & ND & ND & 1.50 & 9.40 & ND & Medium-fine clay & ND \\
\hline $2 \mathrm{H}-4,130-132$ & 13.71 & ND & ND & ND & 1.50 & 9.40 & ND & Medium-fine clay & ND \\
\hline $2 \mathrm{H}-5,130-132$ & 15.21 & ND & ND & ND & 1.50 & 9.40 & ND & Medium-fine clay & ND \\
\hline $2 \mathrm{H}-6,130-132$ & 16.71 & ND & ND & ND & 1.50 & 9.40 & ND & Medium-fine clay & ND \\
\hline $2 \mathrm{H}-7,126-128$ & 18.17 & ND & ND & ND & 1.50 & 9.40 & ND & Medium-fine clay & ND \\
\hline $3 \mathrm{H}-3,147-149$ & 22.59 & ND & ND & ND & 1.50 & 9.40 & ND & Medium-fine clay & ND \\
\hline $3 \mathrm{H}-4,147-149$ & 24.09 & ND & ND & ND & 1.50 & 9.40 & ND & Medium-fine clay & ND \\
\hline $3 \mathrm{H}-5,75-77$ & 24.87 & ND & ND & ND & 1.50 & 9.40 & ND & Medium-fine clay & ND \\
\hline $4 \mathrm{H}-1,140-142$ & 26.41 & ND & ND & ND & 1.50 & 9.40 & ND & Medium-fine clay & ND \\
\hline $4 \mathrm{H}-2,140-142$ & 27.91 & ND & ND & ND & 1.50 & 9.40 & ND & Medium-fine clay & ND \\
\hline $4 \mathrm{H}-3,140-142$ & 29.41 & ND & ND & ND & 1.50 & 9.40 & ND & Medium-fine clay & ND \\
\hline $4 \mathrm{H}-4,80-82$ & 30.31 & ND & ND & ND & 1.50 & 9.40 & ND & Medium-fine clay & ND \\
\hline $4 \mathrm{H}-5,60-62$ & 31.11 & ND & ND & ND & 1.50 & 9.40 & ND & Medium-fine clay & ND \\
\hline $5 \mathrm{H}-1,115-117$ & 35.66 & ND & ND & ND & 1.50 & 9.40 & ND & Medium-fine clay & ND \\
\hline $5 \mathrm{H}-2,115-117$ & 37.16 & ND & ND & ND & 1.50 & 9.40 & ND & Medium-fine clay & ND \\
\hline $5 \mathrm{H}-3,115-117$ & 38.66 & ND & ND & ND & 1.50 & 9.40 & ND & Medium-fine clay & ND \\
\hline $5 \mathrm{H}-4,115-117$ & 40.16 & ND & ND & ND & 1.50 & 9.40 & ND & Medium-fine clay & ND \\
\hline $5 \mathrm{H}-5,115-117$ & 41.66 & ND & ND & ND & 1.50 & 9.40 & ND & Medium-fine clay & ND \\
\hline $5 \mathrm{H}-6,115-117$ & 43.13 & ND & ND & ND & 1.50 & 9.40 & ND & Medium-fine clay & ND \\
\hline $6 \mathrm{H}-1,144-146$ & 45.45 & ND & ND & ND & 1.50 & 9.40 & ND & Medium-fine clay & ND \\
\hline $6 \mathrm{H}-2,144-146$ & 46.95 & ND & ND & ND & 1.50 & 9.40 & ND & Medium-fine clay & ND \\
\hline $6 \mathrm{H}-3,144-146$ & 48.45 & ND & ND & ND & 1.50 & 9.40 & ND & Medium-fine clay & ND \\
\hline $6 \mathrm{H}-4,144-146$ & 49.95 & ND & ND & ND & 1.50 & 9.40 & ND & Medium-fine clay & ND \\
\hline $6 \mathrm{H}-5,144-146$ & 51.45 & ND & ND & ND & 1.50 & 9.40 & ND & Medium-fine clay & ND \\
\hline $6 \mathrm{H}-6,144-146$ & 52.95 & ND & ND & ND & 1.50 & 9.40 & ND & Medium-fine clay & ND \\
\hline 7H-1, 135-137 & 54.86 & ND & ND & ND & 1.50 & 9.40 & ND & Medium-fine clay & ND \\
\hline 7H-2, 135-137 & 56.36 & ND & ND & ND & 1.50 & 9.40 & ND & Medium-fine clay & ND \\
\hline 7H-3, 135-137 & 57.87 & ND & ND & ND & 1.50 & 9.40 & ND & Medium-fine clay & ND \\
\hline 7H-4, 135-137 & 59.37 & 0.00 & 6.20 & 93.80 & 1.88 & 9.06 & 0.51 & Medium clay & Moderately well sorted \\
\hline 7H-5, 135-137 & 60.88 & 0.03 & 96.75 & 3.22 & 8.98 & 6.80 & 0.42 & Fine silt & Well sorted \\
\hline $7 \mathrm{H}-6,18-20$ & 61.21 & 0.01 & 2.15 & 97.84 & 1.98 & 8.98 & 0.25 & Medium clay & Very well sorted \\
\hline $7 \mathrm{H}-7,10-12$ & 62.63 & ND & ND & ND & 1.50 & 9.40 & ND & Medium-fine clay & ND \\
\hline $8 \mathrm{H}-3,130-132$ & 67.31 & ND & ND & ND & 1.50 & 9.40 & ND & Medium-fine clay & ND \\
\hline $8 \mathrm{H}-4,130-132$ & 68.81 & 0.02 & 22.94 & 77.04 & 2.54 & 8.62 & 0.82 & Medium clay & Moderately sorted \\
\hline
\end{tabular}

$\mathrm{ND}=$ not detected 
Table T7. Particle size analysis results, Site U1371. (Continued on next page.)

\begin{tabular}{|c|c|c|c|c|c|c|c|c|c|}
\hline \multirow{2}{*}{$\begin{array}{l}\text { Core, section, } \\
\text { interval }(\mathrm{cm})\end{array}$} & \multirow{2}{*}{$\begin{array}{l}\text { Depth } \\
\text { (mbsf) }\end{array}$} & \multicolumn{3}{|c|}{ Particle size (wt\%) } & \multirow{2}{*}{$\begin{array}{l}\text { Mean } \\
(\mu \mathrm{m})\end{array}$} & \multirow{2}{*}{$\begin{array}{l}\text { Mean } \\
(\phi)\end{array}$} & \multirow{2}{*}{$\begin{array}{l}\text { Sorting } \\
(\phi)\end{array}$} & \multirow{2}{*}{$\begin{array}{l}\text { Grain size } \\
\text { (Wentworth) }\end{array}$} & \multirow[b]{2}{*}{ Sorting } \\
\hline & & Sand & Silt & Clay & & & & & \\
\hline \multicolumn{10}{|l|}{ 329-U1371D- } \\
\hline $1 \mathrm{H}-1,135-137$ & 1.36 & 0.17 & 50.67 & 49.16 & 4.14 & 7.92 & 0.98 & Coarse clay & Moderately sorted \\
\hline $1 \mathrm{H}-2,135-137$ & 2.86 & 0.17 & 70.47 & 29.35 & 6.49 & 7.27 & 1.22 & Very fine silt & Poorly sorted \\
\hline $1 \mathrm{H}-3,135-137$ & 4.36 & 0.29 & 55.36 & 44.35 & 4.57 & 7.77 & 1.16 & Coarse clay & Poorly sorted \\
\hline $1 \mathrm{H}-4,135-137$ & 5.86 & 0.07 & 53.46 & 46.47 & 4.49 & 7.80 & 1.14 & Coarse clay & Poorly sorted \\
\hline $2 \mathrm{H}-1,137-139$ & 8.78 & 0.01 & 66.08 & 33.91 & 5.70 & 7.45 & 1.15 & Very fine silt & Poorly sorted \\
\hline $2 \mathrm{H}-2,137-139$ & 10.28 & 0.13 & 61.43 & 38.44 & 5.11 & 7.61 & 1.13 & Very fine silt & Poorly sorted \\
\hline $2 \mathrm{H}-4,137-139$ & 13.28 & 0.14 & 58.12 & 41.74 & 4.86 & 7.68 & 1.18 & Very fine silt & Poorly sorted \\
\hline $2 \mathrm{H}-5,137-139$ & 14.78 & 0.00 & 42.31 & 57.69 & 3.61 & 8.11 & 0.87 & Coarse clay & Moderately sorted \\
\hline $3 \mathrm{H}-1,134-136$ & 18.25 & 0.00 & 63.97 & 36.03 & 5.48 & 7.51 & 1.18 & Very fine silt & Poorly sorted \\
\hline $3 \mathrm{H}-2,134-136$ & 19.75 & 0.00 & 51.94 & 48.06 & 4.18 & 7.90 & 0.99 & Coarse clay & Moderately sorted \\
\hline $3 \mathrm{H}-3,134-136$ & 21.25 & 0.00 & 50.65 & 49.35 & 4.10 & 7.93 & 0.96 & Coarse clay & Moderately sorted \\
\hline $3 \mathrm{H}-4,134-136$ & 22.75 & 0.07 & 76.74 & 23.19 & 7.33 & 7.09 & 1.21 & Very fine silt & Poorly sorted \\
\hline $3 \mathrm{H}-5,134-136$ & 24.25 & 0.00 & 54.63 & 45.37 & 4.76 & 7.71 & 1.14 & Coarse clay & Poorly sorted \\
\hline $3 \mathrm{H}-6,134-136$ & 25.75 & 0.00 & 77.40 & 22.60 & 7.00 & 7.16 & 1.11 & Very fine silt & Poorly sorted \\
\hline $3 \mathrm{H}-7,60-62$ & 26.51 & 0.00 & 49.94 & 50.06 & 4.16 & 7.91 & 0.99 & Coarse clay & Moderately sorted \\
\hline $4 \mathrm{H}-1,140-142$ & 27.81 & 0.05 & 53.27 & 46.68 & 4.36 & 7.84 & 0.99 & Coarse clay & Moderately sorted \\
\hline $4 \mathrm{H}-2,140-142$ & 29.31 & 0.00 & 48.93 & 51.07 & 4.07 & 7.94 & 0.97 & Coarse clay & Moderately sorted \\
\hline $4 \mathrm{H}-3,140-142$ & 30.81 & 0.14 & 60.22 & 39.64 & 5.56 & 7.49 & 1.36 & Coarse clay & Poorly sorted \\
\hline $4 \mathrm{H}-4,140-142$ & 32.31 & 0.15 & 54.97 & 44.87 & 4.65 & 7.75 & 1.18 & Coarse clay & Poorly sorted \\
\hline $4 \mathrm{H}-5,140-142$ & 33.81 & 0.06 & 50.15 & 49.79 & 4.16 & 7.91 & 0.97 & Coarse clay & Moderately sorted \\
\hline $5 \mathrm{H}-2,140-142$ & 38.41 & 0.19 & 55.15 & 44.66 & 4.77 & 7.71 & 1.21 & Coarse clay & Poorly sorted \\
\hline $5 \mathrm{H}-3,140-142$ & 39.91 & 0.02 & 68.43 & 31.54 & 6.29 & 7.31 & 1.22 & Very fine silt & Poorly sorted \\
\hline $5 \mathrm{H}-4,140-142$ & 41.41 & 0.00 & 49.19 & 50.81 & 4.02 & 7.96 & 0.91 & Coarse clay & Moderately sorted \\
\hline $5 \mathrm{H}-5,140-142$ & 42.91 & 0.00 & 58.55 & 41.45 & 4.67 & 7.74 & 1.09 & Very fine silt & Poorly sorted \\
\hline $6 \mathrm{H}-2,130-132$ & 48.21 & 0.00 & 54.59 & 45.41 & 4.51 & 7.79 & 1.00 & Coarse clay & Moderately sorted \\
\hline $6 \mathrm{H}-3,130-132$ & 49.71 & 0.00 & 53.10 & 46.90 & 4.43 & 7.82 & 1.02 & Coarse clay & Poorly sorted \\
\hline $6 \mathrm{H}-4,130-132$ & 51.21 & 0.23 & 74.42 & 25.35 & 7.71 & 7.02 & 1.38 & Very fine silt & Poorly sorted \\
\hline $6 \mathrm{H}-5,130-132$ & 52.71 & 0.05 & 68.76 & 31.19 & 6.08 & 7.36 & 1.22 & Very fine silt & Poorly sorted \\
\hline $6 \mathrm{H}-6,86-88$ & 53.77 & 0.06 & 73.64 & 26.31 & 6.60 & 7.24 & 1.14 & Very fine silt & Poorly sorted \\
\hline 7H-1, 130-132 & 56.21 & 0.00 & 52.65 & 47.35 & 4.35 & 7.84 & 1.05 & Coarse clay & Poorly sorted \\
\hline 7H-2, 130-132 & 57.71 & 0.04 & 83.72 & 16.24 & 9.84 & 6.67 & 1.29 & Fine silt & Poorly sorted \\
\hline 7H-3, 130-132 & 59.21 & 0.00 & 52.53 & 47.47 & 4.24 & 7.88 & 0.89 & Coarse clay & Moderately sorted \\
\hline 7H-4, 130-132 & 60.71 & 0.04 & 70.08 & 29.89 & 6.07 & 7.36 & 1.11 & Very fine silt & Poorly sorted \\
\hline $7 \mathrm{H}-5,130-132$ & 62.21 & 0.16 & 72.74 & 27.10 & 6.86 & 7.19 & 1.28 & Very fine silt & Poorly sorted \\
\hline 7H-6, 130-132 & 63.71 & 0.12 & 50.40 & 49.48 & 4.44 & 7.81 & 1.12 & Coarse clay & Poorly sorted \\
\hline $8 \mathrm{H}-1,131-133$ & 65.72 & 0.06 & 61.34 & 38.60 & 5.25 & 7.57 & 1.19 & Very fine silt & Poorly sorted \\
\hline $8 \mathrm{H}-2,131-133$ & 67.22 & 0.39 & 71.28 & 28.33 & 7.25 & 7.11 & 1.42 & Very fine silt & Poorly sorted \\
\hline $8 \mathrm{H}-3,131-133$ & 68.72 & 0.05 & 69.03 & 30.92 & 6.15 & 7.35 & 1.10 & Very fine silt & Poorly sorted \\
\hline $8 \mathrm{H}-4,131-133$ & 70.22 & 0.00 & 55.21 & 44.79 & 5.17 & 7.60 & 1.24 & Coarse clay & Poorly sorted \\
\hline $8 \mathrm{H}-5,131-133$ & 71.72 & 0.00 & 61.89 & 38.11 & 5.26 & 7.57 & 1.11 & Very fine silt & Poorly sorted \\
\hline $9 \mathrm{H}-1,130-132$ & 75.21 & 0.08 & 66.36 & 33.56 & 6.63 & 7.24 & 1.28 & Coarse clay & Poorly sorted \\
\hline $9 \mathrm{H}-2,130-132$ & 76.71 & 0.10 & 44.65 & 55.25 & 4.44 & 7.81 & 1.04 & Coarse clay & Poorly sorted \\
\hline $9 \mathrm{H}-3,130-132$ & 78.21 & 0.00 & 76.10 & 23.90 & 7.75 & 7.01 & 1.22 & Very fine silt & Poorly sorted \\
\hline $9 \mathrm{H}-4,130-132$ & 79.71 & 0.01 & 58.52 & 41.47 & 5.58 & 7.49 & 1.26 & Coarse clay & Poorly sorted \\
\hline $9 \mathrm{H}-5,130-132$ & 81.21 & 0.02 & 53.17 & 46.81 & 4.67 & 7.74 & 1.14 & Coarse clay & Poorly sorted \\
\hline $9 \mathrm{H}-6,98-100$ & 82.39 & 0.16 & 55.89 & 43.95 & 5.30 & 7.56 & 1.32 & Coarse clay & Poorly sorted \\
\hline $9 \mathrm{H}-7,98-100$ & 83.39 & 0.00 & 58.42 & 41.58 & 5.29 & 7.56 & 1.19 & Coarse clay & Poorly sorted \\
\hline $10 \mathrm{H}-1,130-132$ & 84.71 & 0.06 & 59.39 & 40.55 & 5.06 & 7.63 & 1.04 & Coarse clay & Poorly sorted \\
\hline $10 \mathrm{H}-2,130-132$ & 86.21 & 0.00 & 51.37 & 48.63 & 4.27 & 7.87 & 0.98 & Coarse clay & Moderately sorted \\
\hline $10 \mathrm{H}-3,130-132$ & 87.71 & 0.00 & 47.09 & 52.91 & 4.24 & 7.88 & 1.12 & Coarse clay & Poorly sorted \\
\hline $10 \mathrm{H}-4,130-132$ & 89.21 & 0.05 & 51.15 & 48.80 & 4.51 & 7.79 & 1.08 & Coarse clay & Poorly sorted \\
\hline $10 \mathrm{H}-5,130-132$ & 90.71 & 0.11 & 43.53 & 56.35 & 3.67 & 8.09 & 0.89 & Coarse clay & Moderately sorted \\
\hline $10 \mathrm{H}-6,130-132$ & 92.21 & 0.27 & 54.38 & 45.35 & 4.88 & 7.68 & 1.20 & Coarse clay & Poorly sorted \\
\hline $11 \mathrm{H}-1,130-132$ & 94.21 & 0.00 & 63.00 & 37.00 & 5.29 & 7.56 & 1.08 & Very fine silt & Poorly sorted \\
\hline $11 \mathrm{H}-2,130-132$ & 95.71 & 0.08 & 68.68 & 31.24 & 6.13 & 7.35 & 1.23 & Very fine silt & Poorly sorted \\
\hline $11 \mathrm{H}-3,130-132$ & 97.21 & 0.00 & 55.72 & 44.28 & 5.27 & 7.57 & 1.21 & Coarse clay & Poorly sorted \\
\hline $11 \mathrm{H}-4,130-132$ & 98.71 & 0.17 & 56.06 & 43.77 & 5.46 & 7.52 & 1.33 & Coarse clay & Poorly sorted \\
\hline $11 \mathrm{H}-5,130-132$ & 100.22 & 0.07 & 58.22 & 41.71 & 5.62 & 7.48 & 1.36 & Coarse clay & Poorly sorted \\
\hline $11 \mathrm{H}-6,130-132$ & 101.72 & 0.05 & 48.61 & 51.34 & 4.74 & 7.72 & 1.31 & Coarse clay & Poorly sorted \\
\hline $12 \mathrm{H}-1,126-128$ & 103.67 & 0.00 & 61.17 & 38.83 & 4.94 & 7.66 & 1.10 & Very fine silt & Poorly sorted \\
\hline $12 \mathrm{H}-2,126-128$ & 105.17 & 0.00 & 54.47 & 45.53 & 4.79 & 7.70 & 1.16 & Coarse clay & Poorly sorted \\
\hline $12 \mathrm{H}-3,126-128$ & 106.67 & 0.00 & 47.94 & 52.06 & 4.07 & 7.94 & 0.98 & Coarse clay & Moderately sorted \\
\hline $12 \mathrm{H}-4,126-128$ & 107.95 & 0.00 & 37.50 & 62.50 & 3.45 & 8.18 & 0.84 & Coarse clay & Moderately sorted \\
\hline $12 \mathrm{H}-5,126-128$ & 109.45 & 0.00 & 30.80 & 69.20 & 3.67 & 8.09 & 0.35 & Coarse clay & Well sorted \\
\hline $12 \mathrm{H}-6,126-128$ & 110.95 & 0.03 & 45.63 & 54.34 & 3.98 & 7.97 & 1.07 & Coarse clay & Poorly sorted \\
\hline $13 \mathrm{H}-1,120-122$ & 113.11 & ND & ND & ND & 1.50 & 9.40 & ND & Medium-fine clay & ND \\
\hline $13 \mathrm{H}-2,120-122$ & 114.61 & ND & ND & ND & 1.50 & 9.40 & ND & Medium-fine clay & ND \\
\hline
\end{tabular}


Table T7 (continued).

\begin{tabular}{|c|c|c|c|c|c|c|c|c|c|}
\hline \multirow{2}{*}{$\begin{array}{l}\text { Core, section, } \\
\text { interval }(\mathrm{cm})\end{array}$} & \multirow{2}{*}{$\begin{array}{l}\text { Depth } \\
\text { (mbsf) }\end{array}$} & \multicolumn{3}{|c|}{ Particle size (wt\%) } & \multirow{2}{*}{$\begin{array}{c}\text { Mean } \\
(\mu \mathrm{m})\end{array}$} & \multirow{2}{*}{$\begin{array}{c}\text { Mean } \\
(\phi)\end{array}$} & \multirow{2}{*}{$\begin{array}{l}\text { Sorting } \\
(\phi)\end{array}$} & \multirow{2}{*}{$\begin{array}{c}\text { Grain size } \\
\text { (Wentworth) }\end{array}$} & \multirow[b]{2}{*}{ Sorting } \\
\hline & & Sand & Silt & Clay & & & & & \\
\hline $13 \mathrm{H}-3,120-122$ & 116.11 & ND & ND & ND & 1.50 & 9.40 & ND & Medium-fine clay & ND \\
\hline $13 \mathrm{H}-4,120-122$ & 117.61 & ND & ND & ND & 1.50 & 9.40 & ND & Medium-fine clay & ND \\
\hline $13 \mathrm{H}-5,120-122$ & 119.11 & ND & ND & ND & 1.50 & 9.40 & ND & Medium-fine clay & ND \\
\hline $13 \mathrm{H}-6,118-120$ & 120.59 & ND & ND & ND & 1.50 & 9.40 & ND & Medium-fine clay & ND \\
\hline $14 \mathrm{H}-1,135-137$ & 122.76 & ND & ND & ND & 1.50 & 9.40 & ND & Medium-fine clay & ND \\
\hline $14 \mathrm{H}-2,135-137$ & 124.26 & ND & ND & ND & 1.50 & 9.40 & ND & Medium-fine clay & ND \\
\hline $14 \mathrm{H}-3,135-137$ & 125.76 & 0.00 & 53.06 & 46.94 & 4.60 & 7.77 & 1.18 & Coarse clay & Poorly sorted \\
\hline
\end{tabular}

ND $=$ not detected 